\title{
LOS DIMINUTIVOS ORGÁNICOS EN LA OBRA DE TERESA DE ÁVILA
}

Elena Carpi, antonina Saba, Manuela Sassi Istituto di Linguistica Computazionale del C.N.R. di Pisa

Nuestro objetivo es mostrar, mediante un caso concreto, cómo en los últimos años la tecnología informática ha conferido un notable impulso a la lingüística aplicada, modificando muchos de los presupuestos técnicos y metodológicos que antes constituían la base de los estudios lingüísticos y literarios.

En consecuencia, los medios informáticos, que en sus comienzos parecían dirigidos al uso exclusivo de las disciplinas científicas, se han convertido en un instrumento cotidiano, vistas sus aplicaciones que van del word processing o tratamiento de textos, que todo el mundo emplea para escribir cartas personales, al tratamiento automático del lenguaje a la Inteligencia Artificial, sin olvidar los usos didácticos y también los trabajos lexicográficos, la compilación de diccionarios y la «manipolación» de amplios corpora textuales.

Nos proponemos, pues, describir la investigación desarrollada sobre el corpus de Teresa de Ávila'; el corpus, analizado y lematizado en su totalidad

' La empresa, iniciada por el profesor Guido Mancini, surgió como fruto de la colaboración entre la Universidad de Pisa y el Instituto de Lingüística Computacional del Consejo Nacional de Investigación de Pisa, y tiene el objetivo de elaborar un diccionario teresiano. 
con la ayuda de un analizador automático ${ }^{2}$, se presenta con un sistema de interrogación de fácil acceso incluso para los usuarios más reacios. El amplio abanico de posibilidades de uso ofrecidas por un corpus así organizado, se puede intuir con facilidad y permite una verificación lingüística precisa de algunos juicios y «prejuicios» sobre la obra de la Santa abulense.

Como ejemplo de uso de este instrumento en el corpus de Santa Teresa, proponemos el análisis del empleo de las formas diminutivas, un aspecto de la prosa teresiana considerado a menudo como efecto de la espontaneidad femenina y de la absoluta falta de elaboración estilística que caracterizarían la obra de la Santa ${ }^{3}$.

A pesar de la nueva tendencia que desde algún tiempo está revisando este juicio ${ }^{4}$, el enlace entre espontaneidad, femineidad e «ignorancia» persiste. De allí deriva la causa del frecuente énfasis en el uso teresiano del diminutivo, considerado una de las señales más importantes de la «escritura femenina».

No obstante cuanto se afirma en los estudios de Mancini y García de la Concha ${ }^{5}$, se considera «abundante» el diminutivo teresiano ${ }^{6}$, sin que se especifique nunca respecto a qué tipo de prosa se asume como punto de referencia. Si se tiene que comparar, a nuestro parecer hay que hacerlo entre la prosa de

2 Morfsin (Analizador morfosintáctico de la lengua espanola), ha sido realizado por A. Saba, D. Rarti, M. N. Catarsi, G. Cappelli, en Pisa en el Instituto de Lingürstica Computacional del Consejo Nacional de la Investigación.

${ }^{3}$ Limitaremos la cita bibliográfica de este tipo de juicios a Don Ramón Menéndez Pidal como ejemplo de la mayoría de los críticos sucesivos, el cual sostiene que rotra causa de la indomable espontaneidad teresiana es la improvisación llevada a grado extremo*, que "Teresa habla por escritox, y que «prescinde por completo de todo uso estilistico», en La Lengua de Cristóbal Colón, Buenos Aires, 1947.

4 Por ejemplo: Fernando Lázaro Carreter, «Santa Teresa de Jesús, escritoraw, en Actas del Congreso Internacional Teresiano, Salamanca, 4-7 octubre 1982, Salamanca, 1983. Victor García de la Concha, El arte literario de Santa Teresa, Barcelona, 1978.

S En Espressioni letterarie dell'insegnamento di Teresa de Avila, Modena, 1955, Mancini subraya como la Santa abulense no hacia «un uso eccessivo* del diminutivo. Pero Mancini no indica el número de los diminutivos obra por obra y no son especificados los criterios de individuación (sí, por ejemplo, tales cantidades comprenden también los términos lexicalizados). García de la Concha, con referencia a este estudio de Mancini (Cfr. nota 3), confirma sus datos que esin duda relativizan las afirmaciones habituales..

- El mismo Menéndez Pidal, Cfr. nota 2, afirma que Teresa de Ávila esentía una propensión irreprimible hacia esta forma gramatical ; Emilio Núfiez Femández in El diminutivo. Historia y funciones en el espafiol clásico y modemo, Madrid, 1973: «el diminutivo preciso, amoroso, con el que tiene toda la frase de la más deliciosa feminidad... Es el diminutivo una de las formas gramaticales preferidas por Santa Teresa*; Alison Weber, «Teresa's «delicious» diminutives: pragmatics and style in Camino de Perfección, in Journal of Hispanic Philology, 10, 1985-1986: "In this paper $I$ would like to concentrate on an aspect of Teresa's writing... her abundant use of diminutivesw. 
Teresa de Ávila y la de los demás místicos y escritores de obras religiosas, prescindiendo del estilo elegante cuyos principios Herrera fija ${ }^{7}$ a comienzos del siglo XVI en las Anotaciones a las obras de Garcilaso ${ }^{8}$.

$\mathrm{Si}$, por ejemplo, confrontamos las Poesías de la Santa abulense con la obra poética de Fray Luis de León, cuyas concordancias están editadas por Vicente Sabido encontramos sólo tres diminutivos en la primeras y uno en las de Fray Luis, dato que, respecto al número total de palabras empleadas por los dos escritores, evidencia un análogo empleo de la forma diminutiva.

De la verificación del corpus lematizado se extrae que el porcentaje de diminutivos presentes en las obras en relación a las palabras no autoriza ni juicios de abundancia referidos a la forma diminutiva, ni de casualidad y arbitrariedad: existen 78 diminutivos en la Vida, 48 en el Camino de Perfección, 48 en las Fundaciones, 61 en las Moradas del Castillo Interior, 191 en las Cartas, 24 en los Conceptos del Amor de Dios, y 30 en las demás Obras Menores: en total 430 en todo el corpus.

\begin{tabular}{|l|c|c|c|c|}
\hline \multicolumn{1}{|c|}{ Obras $^{10}$} & $\begin{array}{c}\text { Ocurrencias de } \\
\text { diminutivos }^{11}\end{array}$ & $\begin{array}{c}\text { Formas de } \\
\text { diminutivos }\end{array}$ & $\begin{array}{c}\text { Lemas de } \\
\text { diminutivos }\end{array}$ & $\begin{array}{c}\text { Ocurrencias de } \\
\text { cada obra }\end{array}$ \\
\hline VID & 78 & 53 & 42 & 112.200 \\
CAM & 48 & 30 & 22 & 52.038 \\
FUN & 48 & 34 & 32 & 74.752 \\
CAR & 191 & 113 & 79 & 283.340 \\
MOR & 61 & 40 & 30 & 59.124 \\
REL & 11 & 10 & 10 & 41.605 \\
CON & 24 & 18 & 16 & 15.930 \\
EXC & 2 & 2 & 2 & 7.357 \\
COS & 1 & 1 & 1 & 7.266 \\
\hline
\end{tabular}

7 Como Menendez Pidal, p. 134 ib., y Núñez, p. 192 ib.

- Obras de Garci Lasso de la Vega con anotaciones de Fernando de Herrera... En Sevilla, por Alonso de la Barrera, ano de 1580, p. 554. Edició en Facsimile de Antonio Gallego Morell, Clásicos Hispánicos, C.S.I.C., Madrid, 1973: «la lengua Toscana está llena de deminutos... la nuestra no los recibe si no con mucha dificultad, i mui pocas vezes". Esta afirmación, a prueba de la importancia del tipo di lengua que se asuma como referencia, es contradicha por la gramática de Lovaino (1555), que denuncia una presencia abundante de esta forma en la lengua española a lo largo del siglo XVI.

- Vicente Sabido, Concordancias de la poesta original de Fray Luis de León, Universidad de Granada, 1992.

to VEase la tabla de correspondencia de los códigos al anexo 2.

1 Véase la tabla de las formas diminutivas divididas por obra al anexo 1. 


\begin{tabular}{|l|c|c|c|c|}
\hline Obras & $\begin{array}{c}\text { Ocurrencias de } \\
\text { diminutivos }\end{array}$ & $\begin{array}{c}\text { Formas de } \\
\text { diminutivos }\end{array}$ & $\begin{array}{c}\text { Lemas de } \\
\text { diminutivos }\end{array}$ & $\begin{array}{c}\text { Ocurrencias de } \\
\text { cada obra }\end{array}$ \\
\hline MOD & 2 & 2 & 2 & 6.171 \\
POE & 6 & 3 & 3 & 4.705 \\
APU & 8 & 5 & 4 & 4.429 \\
RES & 0 & 0 & 0 & 1.380 \\
VEJ & 0 & 0 & 0 & 863 \\
Totales & 480 & & & 671.160 \\
\hline
\end{tabular}

Tabla 1: Formas diminutivas de cada obra con relativos lemas y ocurrencias totales.

En la tabla 1 se muestra el número de diminutivos en cada obra junto al número total de palabras; de esa relación se puede extraer un porcentaje individual de diminutivos en cada una de las obras, como se evidencia en el diagrama que sigue. (Gráfico 1).

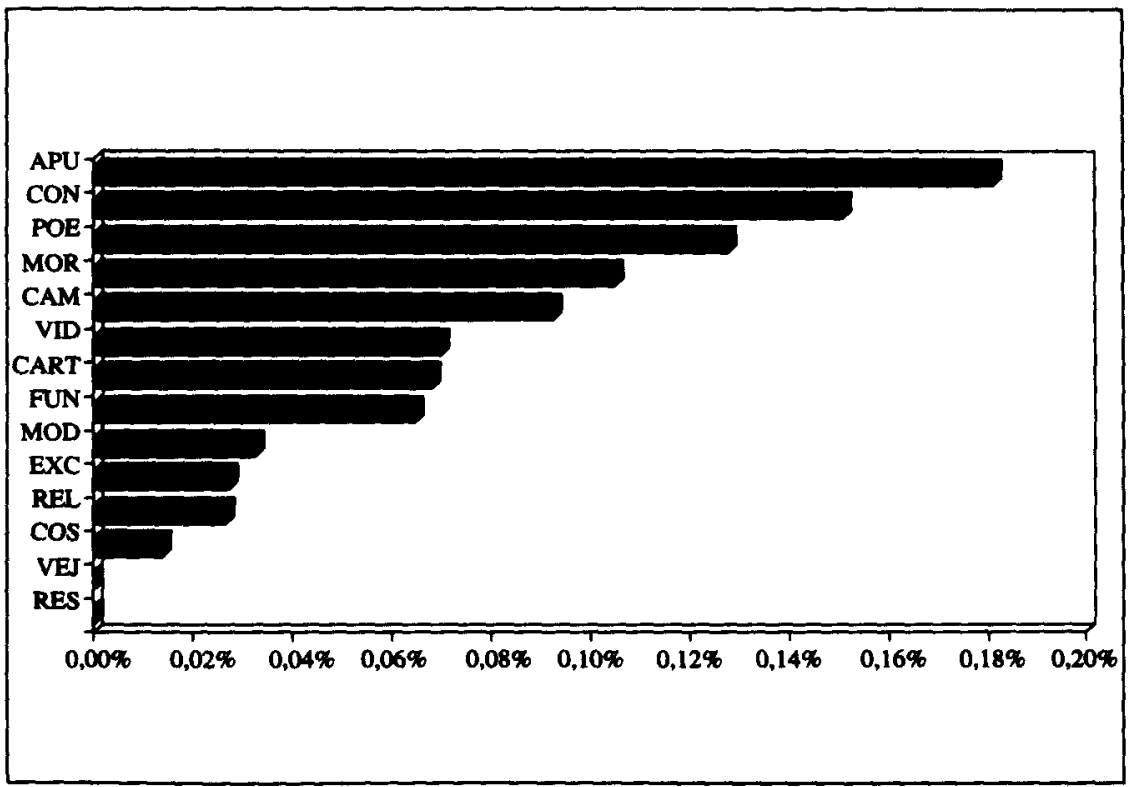

Gráfico 1: Formas diminutivas de cada obra en relación a las ocurrencias totales. 
El número final de los lemas es todavía menor, 166, debido a la repetición de las formas, mientras el porcentaje absoluto de frecuencia de la forma diminutiva en todo el corpus lematizado es de $0,07 \%$.

Por ejemplo, si en los Conceptos aparecen 24 diminutivos, esa cifra constituye un $0,15 \%$, aproximado por defecto, respecto al total de 15930 palabras que tiene esta obra. Mientras que en la Vida los 78 diminutivos constituyen un $0,07 \%$.

Se trata en ambos casos de porcentajes individuales. Dichos datos en relación al total del corpus teresiano constituyen lo que denominamos densidad. Asi el 0,15\% de los Conceptos, que resultaría de la lectura de la tabla 2, aparece como un $16 \%$, aproximado por exceso, de densidad del total y el $0,07 \%$ de la Vida aparece como un 7\%.

En el diagrama circular de sectores queda sintetizado el porcentaje de uso de los diminutivos empleados por la Santa, divididos por obra. (Gráfico 2)

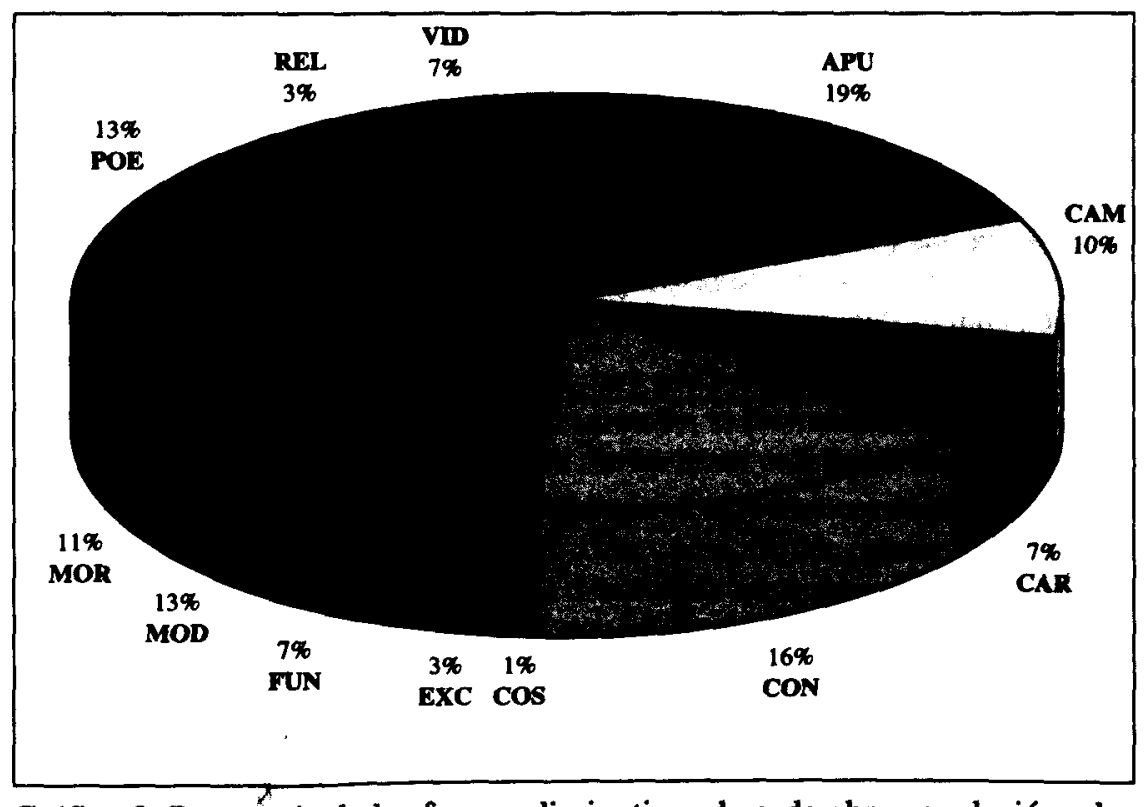

Grafico 2: Porcentaje de las formas diminutivas de cada obra en relación a las ocurrencias totales.

Como se puede notar, el porcentaje de diminutivos varía de una obra a otra: por esta razón es muy difícil establecer criterios de variación, pero es posible presentar hipótesis sobre la disminución o el aumento fundándose sobre el carácter de las diferentes obras. 
En un primer grupo de obras, Constituciones, Modo de Visitar los Conventos, Relaciones, este porcentaje varía del $1 \%$ al $3 \%$ : la primera es el estatuto de la Orden, la segunda contiene avisos dirigidos a los prelados encargados de la dirección espiritual de las monjas de los conventos carmelitas, la tercera reune apuntes personales sobre diferentes experiencias y acontecimientos. Fuera de algunas excepciones, la Santa adécua su prosa al contenido y a la función de estos escritos, usando un lenguaje eficaz y realista en el que la forma diminutiva encuentra poco lugar.

De este primer grupo hemos separado las Exclamaciones del alma a Dios, a pesar de un porcentaje análogo: en este caso la Santa abulense escoge como vehículo para expresarse la forma retórica de la amplificatio.

Las Exclamaciones presentan una compleja estructura en su composición interna y en el periodo, construído por pasajes sucesivos de imágenes contrapuestas entre sí, interiormente animado por una incesante superación dialéctica. $A$ causa de estas características, la Santa abulense no necesita aquí emplear diminutivos, a pesar del inflamado desahogo místico que caracteriza esta obra.

Siguen las Cartas de la Santa, en las cuales el porcentaje de diminutivos es solo del 7\%, a pesar del tipo de lenguaje que, por ser más familiar, podría sufrir un sensible aumento de esta forma, y dos de los textos mayores de Teresa de Ávila, las Fundaciones y la Vida, en los que el porcentaje varía del 7\% al $10 \%$. Subrayamos un uso restringido del diminutivo, que a nuestro parecer también aquí es debido al género de contenido.

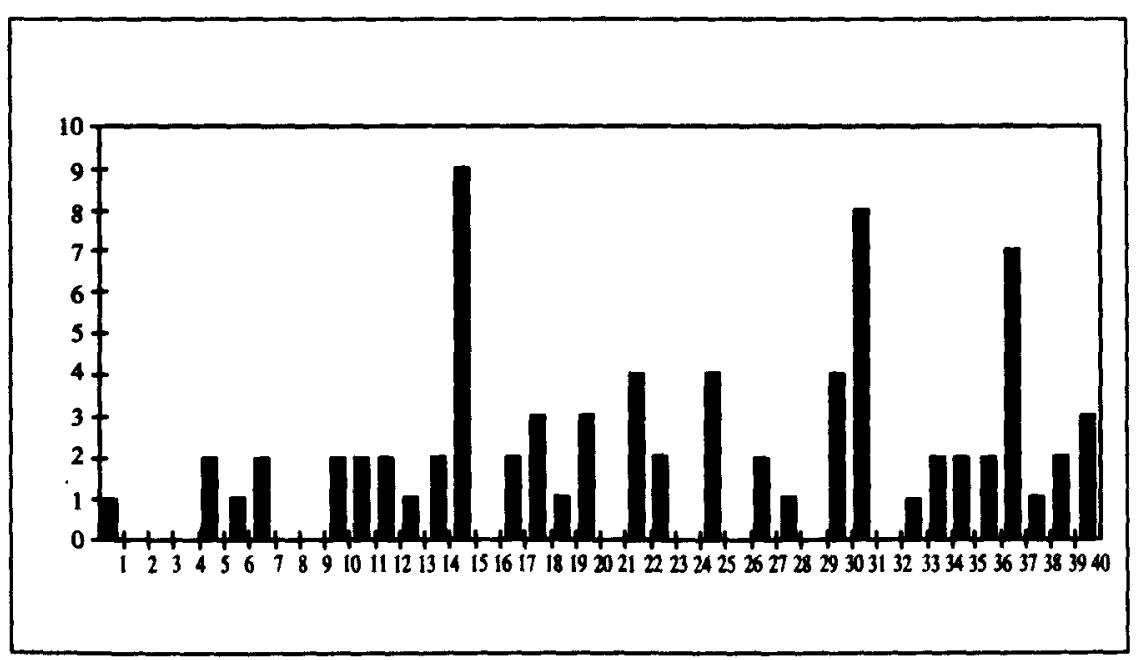

Gráfico 3: Distribución de los diminutivos en los capítulos de la Vida. 
Con esta finalidad hemos examinado la distribución de los diminutivos en la Vida, la obra más extensa y donde los diminutivos son bastante frecuentes. En 11 capítulos no hay ninguno, en 7 hay sólo 1, en 13 hay 2, en 3 hay 3, en 4 hay 3 , mientras en los capítulos 15,31 y 37 se advierte un considerable incremento del número de los diminutivos. (Gráfico 3).

En el capítulo 15 los 8 diminutivos ${ }^{12}$ están todos referidos al estado de pobreza espiritual del alma que empieza el camino de la oración. Tambien en el capítulo 31 los diminutivos ${ }^{13}$ se refieren a la humildad y a la escasa consideración de sí mismos que hay que sentir en relación a Dios.

En el 37, en fín, están presentes 7 diminutivos ${ }^{14}$ y siguen refiriéndose a ideas análogas a las de los capítulos analizados anteriormente.

La Santa emplea los mismos vocablos para describir situaciones espirituales afines, y usa el diminutivo de manera homogénea para acentuar el valor despectivo de palabras elejidas en función de la misma situación.

El porcentaje de diminutivos aumenta en el Camino de Perfección, en los Apuntes, en las Moradas del Castillo Interior y en los Conceptos del Amor de Dios pero con tal proposito se tienen que hacer algunas consideraciones. Entre la primera y la segunda redacción del Camino de Perfección la Santa abulense interviene eliminando 26 formas diminutivas, por razones doctrinales o estilísticas, intervención que nos parece muy significativa acerca de la «voluntad de estilo» de la Santa, cuya espontaneidad no significa necesariamente descuido estilístico.

En lo que concierne a los Apuntes, hay que subrayar que los diminutivos, que se encuentran todos en el mismo fragmento, la Instrucción de la madre Teresa de Jesús para la Madre priora de Soria, están casi todos lexicalizados.

Nos parece pues del todo razonable que el mayor porcentaje de diminutivos se encuentre en las Moradas y los Conceptos. En las primeras el dato autobiografico se profundiza, y la Santa describe la misteriosa relación del alma con su Dios a traves de la oración y el estado místico de unión. Los últimos son el comentario al Cantar de los Cantares de Salomón, y contienen meditaciones personales y desahogos motivados por el texto b́blico. Los diminutivos empleados por la Santa tienen en estos casos un valor afectivo evidente, junto al reductivo referido a la insuficiencia y pequeriez del alma que vive la experiencia mística.

12 Los lemas son centella, consideración, pequeño, paja, pobre y tanto. Se utiliza el carácter en negrita para los lemas que se encuentren en más de uno de los tres capitulos.

${ }_{13}$ Los lemas son negro, temor, arena, hormiga, paja, pobre, poco, tance.

14 Los lemas son pobre, poco, tanto, palo. 
Dejando a un lado el criterio exclusivamente numérico, hay que notar que el uso reiterado de los diminutivos, incluso de los que poseen un aparente valor afectivo, y el hecho que se empleen siempre en relación a la misma simbología, atenua sensiblemente su efecto de disminución o de afectividad.

Tomamos como ejemplo los diferentes tipos de diminutivos que derivan de mariposa: mariposilla y mariposica están presentes 6 veces, mariposita 3 . la palabra mariposa está sostituida casi completamente por sus diminutivos, y como se notará en los ejemplos que siguen, el valor afectivo-reductivo de sus diminutivos queda muy atenuado:

Si habrán bastado todas esas mercedes que ha hecho el Esposo a el alma para que la palomilla u mariposilla esté satisfecha...

Moradas 6, cap. 11

Conforme a mi ingenio porné una comparación; después diremos más de esta mariposica, que no para, porque no halla su verdadero reposo.

Moradas 5, cap. 4

...que no parece sino de estas maripositas de las noches, importunas y desasosegadas...

Vida, cap.17

Estos ejemplos introducen el tema de la variedad de los diminutivos a los cuales la Santa recurre mayormente: el diagrama circular de sectores que sigue expone los porcentajes de los diferentes tipos de diminutivos en referencia al total de las formas diminutivas. (Gráfico 4)

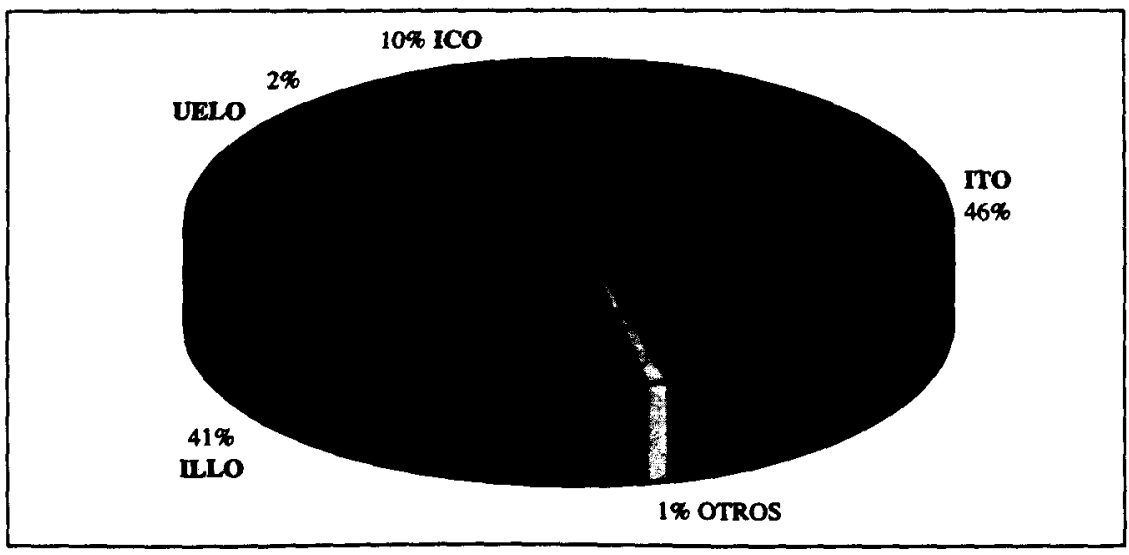

Gráfico 4: Distribución de los tipos de diminutivos en relación a los diminutivos totales. 
Illo e ito son evidentemente los sufijos empleados con mayor frecuencia, $46 \%$ el primero y $41 \%$ el segundo, mientras los demás están presentes en porcentajes mucho más bajos. De un análisis de los contextos se nota que el sufijo illo es empleado con valor peyorativo, pero que puede tener también cierta complacencia afectiva:

aparejado un reino que no tiene fin por unos trabajillos envueltos en mil contentos...

Vida, cap. 31

«De qué te afliges, pecadorcilla? Yo no soy tu Dios? Relaciones, cap. 21

Puede además ser empleado con la intención de expresar escasa consideración para el objeto de que se está hablando:

Pues hacer mucho ruido al caerse casa de trece pobrecillas no es bien...

Vida, cap. 2

El sufijo ico, empleado con el mismo valor de ito, es al contrario indicio de la máxima afectividad. También aquí presentamos unos contextos:

Pues tornemos a nuestra palomica.

Pues adónde ira la pobrecica?

Las reliquias de los Santos Pastorcicos,
Moradas 5, cap. 3

Moradas 5, cap. 2

Cartas 345.01

La tabla 2 señala la frecuencia de los diferentes sufijos en las distintas obras.

\begin{tabular}{|l|c|r|r|r|r|r|}
\hline \multicolumn{1}{|c|}{ OBRAS } & OTROS & ICO & LLO & ITO & UELO & TOTAL \\
\hline APU & 0 & 1 & 7 & 0 & 0 & 8 \\
CAM & 0 & 1 & 13 & 33 & 1 & 48 \\
CAR & 1 & 20 & 80 & 82 & 8 & 191 \\
CON & 0 & 1 & 13 & 10 & 0 & 24 \\
COS & 0 & 0 & 1 & 0 & 0 & 1 \\
EXC & 0 & 0 & 1 & 1 & 0 & 2 \\
FUN & 1 & 2 & 21 & 23 & 1 & 48 \\
MOD & 0 & 0 & 2 & 0 & 0 & 2 \\
MOR & 1 & 13 & 25 & 22 & 0 & 61 \\
POE & 0 & 0 & 6 & 0 & 0 & 6 \\
\hline
\end{tabular}




\begin{tabular}{|l|c|r|r|r|r|r|}
\hline \multicolumn{1}{|c|}{ OBRAS } & OTROS & ICO & ILLO & ITO & UELO & TOTAL \\
\hline REL & 0 & 2 & 6 & 3 & 0 & 11 \\
VID & 0 & 8 & 23 & 47 & 0 & 78 \\
TOTAL & 3 & 48 & 198 & 221 & 10 & 480 \\
\hline
\end{tabular}

Tabla 2: Frecuencia de los tipos de diminutivos en cada obra.

El sufijo illo está presente en porcentaje abundante en las Cartas (80), en las Moradas (25)y en las Fundaciones (21), mientras ito se encuentra sobre todo en las Cartas, en la Vida y en el Camino de Perfección.

De esta rápida descripción se evidencia como las elecciones lingúísticas de Teresa de Avila se demuestran consonantes con las de su época, en la cual se afirma el uso de los sufijos ico e ito.

Nuestro análisis, que ha aprovechado las posibilidades ofrecidas por el corpus lematizado, ha puesto de relieve el empleo no casual de las formas diminutivas presentes en la prosa teresiana.

A pesar de los juicios de descuido, y de la misma polémica de la escritora abulense contra los «letrados», nos parece que la forma que Teresa de Ávila elije para comunicar la experiencia mística resulta inseparable de su propio contenido, y desmiente los que quieren ver en los tratados teresianos sólo la manifestación devocional de una mujer apasionada, negando a la autora cualquiera preocupación estilística.

Anexo 1

Tabla de las formas diminutivas divididas por obra

\begin{tabular}{|l|c|c|c|c|c|c|c|c|c|c|c|c|c|}
\hline \multicolumn{1}{|c|}{ FORMAS } & APU & CAM & CAR & CON & COS & EXC & FUN & MOD & MOR & POE & REL & VID & TOT \\
\hline airecito & - & - & - & - & - & - & - & - & - & - & - & 1 & 1 \\
amaditas & - & - & 1 & - & - & - & - & - & - & - & - & - & 1 \\
angelito & - & - & 6 & - & - & - & - & - & - & - & - & - & 6 \\
angelitos & - & - & 2 & - & - & - & - & - & - & - & - & - & 2 \\
aposentico & - & - & 1 & - & - & - & - & - & - & - & - & - & 1 \\
arbotillo & - & - & - & 1 & - & - & - & - & - & - & - & - & 1 \\
arenitas & - & - & - & - & - & - & - & - & - & - & - & 1 & 1 \\
arquilla & - & - & 7 & - & 1 & - & - & - & - & - & - & - & 8 \\
arroyico & - & - & - & - & - & - & - & - & 1 & - & - & - & 1 \\
arrovicos & - & - & - & - & - & - & - & - & 1 & - & - & - & 1 \\
\hline
\end{tabular}


Anexo 1 (Continuación)

Tabla de las formas diminutivas divididas por obra

\begin{tabular}{|c|c|c|c|c|c|c|c|c|c|c|c|c|c|}
\hline FORMAS & APU & CAM & CAR & $\mathrm{CON}$ & $\cos$ & EXC & FUN & MOD & MOR & $\mathrm{POE}$ & REL & VID & TOT \\
\hline 2smillo & - & - & - & - & - & - & - & - & - & - & - & 1 & 1 \\
\hline 2snillos & - & - & - & - & - & - & - & - & - & - & - & 1 & 1 \\
\hline avecica & - & - & - & - & - & - & - & - & - & - & 1 & - & 1 \\
\hline avecita & - & - & - & - & - & - & - & - & - & - & - & 2 & 2 \\
\hline ayditios & - & - & - & - & - & - & - & - & - & - & - & 1 & 1 \\
\hline bendillo & - & - & 2 & - & - & - & - & - & - & - & - & - & 2 \\
\hline bandillos & - & 1 & - & - & - & - & - & - & - & - & - & - & 1 \\
\hline Bernardino & - & - & 1 & - & - & - & - & - & - & - & - & - & 1 \\
\hline blanquilles & - & - & - & - & - & - & 1 & - & - & - & - & - & 1 \\
\hline bolith & - & - & 1 & - & - & - & - & - & - & - & - & - & 1 \\
\hline bonita & - & - & 17 & - & - & - & - & - & - & - & - & - & 17 \\
\hline bonitss & - & - & 1 & - & - & - & - & - & - & - & - & - & 1 \\
\hline bonitos & - & - & - & - & - & - & 1 & - & - & - & - & - & 1 \\
\hline boquilites & - & - & - & - & - & - & - & - & 1 & - & - & - & 1 \\
\hline borriquillos & - & - & 1 & - & - & - & - & - & - & - & - & - & 1 \\
\hline bringuinillos & - & - & 1 & - & - & - & - & - & - & - & - & - & 1 \\
\hline calderica & - & - & 1 & - & - & - & - & - & - & - & - & - & 1 \\
\hline caleuturill & - & - & 1 & - & - & - & - & - & - & - & - & - & 1 \\
\hline calenturitles & - & - & 1 & - & - & - & - & - & - & - & - & - & 1 \\
\hline calorcit & - & - & 1 & - & - & - & - & - & - & - & - & - & 1 \\
\hline camarilla & - & - & - & - & - & - & 2 & - & - & - & - & - & 2 \\
\hline campanillh & - & - & - & - & - & - & 2 & - & - & - & - & - & 2 \\
\hline contarcillo & - & - & 1 & - & - & - & - & - & - & - & 1 & - & 2 \\
\hline capitilla & - & - & 1 & - & - & - & - & - & - & - & - & - & 1 \\
\hline capactillio & - & - & - & - & - & - & - & - & 1 & - & - & - & 1 \\
\hline capnchillos & - & - & - & - & - & - & - & - & 1 & - & - & - & 1 \\
\hline carcetilla & - & - & 1 & - & - & - & - & - & - & - & - & - & 1 \\
\hline casilla & - & - & 4 & - & - & - & 1 & - & - & - & - & - & 5 \\
\hline casita & - & - & 3 & - & - & - & 2 & - & - & - & - & 1 & 6 \\
\hline casitas & - & - & I & - & - & - & - & - & - & - & - & - & 1 \\
\hline castillito & - & 1 & - & - & - & - & - & - & - & - & - & - & 1 \\
\hline celditla & - & - & 1 & - & - & - & - & - & - & - & - & - & 1 \\
\hline centelica & - & 1 & - & - & - & - & - & - & - & - & - & 4 & 5 \\
\hline cerrojillos & - & - & 1 & - & - & - & - & - & - & - & - & - & 1 \\
\hline charquitos & - & 1 & - & - & - & - & - & - & - & - & - & - & 1 \\
\hline
\end{tabular}


Anexo 1 (Continuación)

Tabla de las formas diminutivas divididas por obra

\begin{tabular}{|c|c|c|c|c|c|c|c|c|c|c|c|c|c|}
\hline FORMAS & APU & CAM & CAR & $|\operatorname{coN}|$ & $\cos \mid$ & EXC & FUN & MOD & MOR & POE & REL & VID & TOT \\
\hline chinillas & - & - & - & - & - & - & - & - & 1 & - & - & - & 1 \\
\hline chiquilla & - & - & 1 & - & - & - & - & - & - & - & - & - & 1 \\
\hline chiquillo & - & - & 1 & - & - & - & - & - & - & - & - & - & 1 \\
\hline chiquita & - & 1 & 1 & - & - & - & - & - & - & - & - & - & 2 \\
\hline chiquito & - & - & 1 & - & - & - & - & - & - & - & - & - & 1 \\
\hline cobanillo & - & - & - & - & - & - & - & - & - & 1 & - & - & 1 \\
\hline cocinilh & - & - & - & - & - & - & 1 & - & - & - & - & - & 1 \\
\hline concticas & - & - & - & - & - & - & - & - & - & - & - & 1 & 1 \\
\hline condicioncita & - & - & 1 & - & - & - & - & - & - & - & - & - & 1 \\
\hline considerncioncillas & - & - & - & - & - & - & - & - & - & - & - & 1 & 1 \\
\hline contentillos & - & - & - & - & - & - & - & - & 1 & - & - & - & 1 \\
\hline cordecita & - & - & 1 & - & - & - & - & - & - & - & - & - & 1 \\
\hline corderitos & - & - & - & - & - & - & 1 & - & - & - & - & - & 1 \\
\hline cormatillo & - & - & 1 & - & - & - & - & - & - & - & - & - & 1 \\
\hline corpezuelos & - & 1 & - & - & - & - & - & - & - & - & - & - & 1 \\
\hline cosilh & - & - & 1 & - & - & - & - & - & - & - & - & - & 1 \\
\hline cosiltas & - & 1 & 4 & 3 & - & - & 2 & 1 & 1 & - & - & 2 & 14 \\
\hline cosita & - & 2 & 1 & 1 & - & - & 1 & - & 2 & - & - & - & 7 \\
\hline cosititus & - & 1 & - & - & - & - & - & - & - & - & - & 2 & 3 \\
\hline coverach & - & - & - & - & - & - & 1 & - & - & - & - & - & 1 \\
\hline cracocica & - & - & 1 & - & - & - & - & - & - & - & - & - & 1 \\
\hline cuadernillo & - & - & 3 & - & - & - & - & - & - & - & 1 & - & 4 \\
\hline cuadernillos & - & - & 1 & - & - & - & - & - & - & - & - & - & 1 \\
\hline cuartill & - & - & 1 & - & - & - & - & - & - & - & - & - & 1 \\
\hline cuartillo & - & - & 1 & - & - & - & - & - & - & - & - & - & 1 \\
\hline cuidadito & - & 1 & - & - & - & - & - & - & - & - & - & 1 & 2 \\
\hline determimacioncilla & - & 1 & - & - & - & - & - & - & - & - & - & - & 1 \\
\hline devocioncitas & - & - & - & - & - & - & - & - & - & - & - & 1 & 1 \\
\hline disgustillo & - & - & - & - & - & - & - & - & - & - & - & 1 & 1 \\
\hline Dominguillo & - & - & - & - & - & - & - & - & - & 4 & - & - & 4 \\
\hline donecito & - & - & - & 1 & - & - & - & - & - & - & - & - & 1 \\
\hline Elenita & - & - & 1 & - & - & - & - & - & - & - & - & - & 1 \\
\hline encarceladitita & - & - & - & - & - & - & - & - & - & - & - & 1 & 1 \\
\hline enfermita & - & - & 1 & - & - & - & - & - & - & - & - & - & 1 \\
\hline ermitillas & - & -1 & - & - & -1 & - & 1 & -1 & - & - & - & - & 1 \\
\hline
\end{tabular}


Anexo 1 (Continuación)

Tabla de las formas diminutivas divididas por obra

\begin{tabular}{|c|c|c|c|c|c|c|c|c|c|c|c|c|c|}
\hline FORMAS & APU & САM & CAR & CON & $\cos \mid$ & EXC & FUN & MOD & MOR & $\mathrm{POE}$ & REL & VID & TOT \\
\hline eschvilla & & - & 1 & - & - & - & - & - & - & - & - & - & 1 \\
\hline inita & & - & - & 1 & - & - & - & - & - & - & - & - & 1 \\
\hline faltita & - & - & - & - & - & - & - & - & 1 & - & - & - & 1 \\
\hline Alorecitas & - & - & - & - & - & - & - & - & - & - & - & 2 & 2 \\
\hline fontecicas & & - & - & - & - & - & - & - & - & - & - & 1 & 1 \\
\hline fontecita & - & - & - & - & - & - & - & - & 1 & - & - & - & 1 \\
\hline frailecico & & - & - & - & - & - & 1 & - & - & - & - & - & 1 \\
\hline ilecitos & - & - & 1 & - & - & - & - & - & - & - & - & - & 1 \\
\hline Francisquito & & - & 1 & - & - & - & - & - & - & - & - & - & 1 \\
\hline ganadillo & - & - & - & - & - & - & - & - & - & 1 & - & - & 1 \\
\hline Gomanlito & - & - & 1 & - & - & - & - & - & - & - & - & - & 1 \\
\hline rdillh & - & - & 1 & - & - & - & - & - & - & - & - & - & 1 \\
\hline gorditit & - & - & 1 & - & - & - & - & - & - & - & - & - & 1 \\
\hline grnantillo & - & - & 1 & - & - & - & - & - & - & - & - & - & 1 \\
\hline erritles & & - & - & 1 & - & - & - & - & - & - & - & - & 1 \\
\hline gusanillo & - & 1 & - & 1 & - & 1 & - & - & 2 & - & - & - & 5 \\
\hline guseanitos & - & - & - & - & - & - & - & - & 1 & - & - & - & 1 \\
\hline haveccito & - & - & - & - & - & - & 1 & - & - & - & - & - & 1 \\
\hline bermanita & - & - & 2 & - & - & - & - & - & - & - & - & - & 2 \\
\hline hermosita & & - & 1 & - & - & - & - & - & - & - & - & - & 1 \\
\hline hierbecillas & 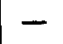 & - & - & - & - & - & - & - & - & - & - & 1 & 1 \\
\hline hormizailh & - & - & - & - & - & - & - & - & - & - & - & 1 & 1 \\
\hline hormiguith & & - & - & - & - & - & - & - & 1 & - & - & - & 1 \\
\hline hornico & - & - & 1 & - & - & - & - & - & - & - & - & - & 1 \\
\hline hormillo & - & - & 1 & - & - & - & - & - & - & - & - & - & 1 \\
\hline hornito & & - & 1 & - & - & - & - & - & - & - & - & - & 1 \\
\hline huertecillo & - & - & - & - & - & - & 1 & - & - & - & - & - & 1 \\
\hline idoilillo & & - & - & - & - & - & - & - & - & - & - & 2 & 2 \\
\hline sita & & - & - & - & - & - & 2 & - & - & - & - & - & 2 \\
\hline ratillh & & - & 1 & - & - & - & - & - & - & - & - & - & 1 \\
\hline Isabclita & - & - & 6 & - & - & - & - & - & - & - & - & - & 6 \\
\hline rita & - & - & 1 & - & - & - & - & - & - & - & - & - & 1 \\
\hline guilla & - & - & 2 & - & - & - & - & - & - & - & - & - & 2 \\
\hline Juanico & - & - & 1 & - & - & - & - & - & - & - & - & - & 1 \\
\hline labradorillt & - & - & -1 & 1 & - & - & -1 & - & -1 & - & -1 & - & 1 \\
\hline
\end{tabular}


Anexo 1 (Continuación)

Tabla de las formas diminutivas divididas por obra

\begin{tabular}{|c|c|c|c|c|c|c|c|c|c|c|c|c|c|}
\hline FORMAS & APU & CAM & CAR & CON & $\cos$ & EXC & FUN & MOD & MOR & POE & REL & VID & TOT \\
\hline bradorcita & - & - & - & - & - & - & 1 & - & - & - & - & - & 1 \\
\hline lagartijillos & - & - & - & - & - & - & - & - & 1 & - & - & - & 1 \\
\hline lagrimitles & - & - & - & - & - & - & - & - & - & - & - & 1 & 1 \\
\hline lamparilla & 1 & - & - & - & - & - & - & - & - & - & - & - & 1 \\
\hline lengiecill & - & - & - & - & - & - & - & - & - & - & - & 1 & 1 \\
\hline Leonorica & - & - & 1 & - & - & - & - & - & - & - & - & - & 1 \\
\hline librico & - & - & - & - & - & - & 1 & - & - & - & - & - & 1 \\
\hline librillo & - & - & 4 & - & - & - & - & - & - & - & 1 & - & 5 \\
\hline Ilarecica & 1 & - & - & - & - & - & - & - & - & - & - & - & 1 \\
\hline logancillo & - & - & 1 & - & - & - & 3 & - & - & - & - & - & 3 \\
\hline lugarcillos & - & - & 1 & - & - & - & - & - & - & - & - & - & 1 \\
\hline legarillos & - & - & 1 & - & - & - & - & - & - & - & - & - & 1 \\
\hline machaedo & - & - & 2 & - & - & - & - & - & - & - & - & - & 2 \\
\hline maderillo & - & - & - & - & - & - & - & - & - & - & - & 1 & 1 \\
\hline malectllos & - & 2 & - & - & - & - & - & - & - & - & - & - & 2 \\
\hline mariposica & - & - & - & - & - & - & - & - & 6 & - & - & - & 6 \\
\hline mariposilla & - & - & - & - & - & - & - & - & 5 & - & - & - & 5 \\
\hline mariposita & - & - & - & - & - & - & - & - & 2 & - & - & - & 2 \\
\hline maripocitas & - & - & - & - & - & - & - & - & - & - & - & 1 & 1 \\
\hline membrillos & - & - & 4 & - & - & - & - & - & - & - & - & - & 4 \\
\hline mocito & - & - & 1 & - & - & - & - & - & - & - & - & - & 1 \\
\hline mocitos & - & - & 2 & - & - & - & - & - & - & - & - & - & 2 \\
\hline menjith & - & - & 1 & - & - & - & - & - & - & - & - & - & 1 \\
\hline monjillas & - & - & 1 & - & - & - & - & - & - & - & - & - & 1 \\
\hline morjuch & - & - & 1 & - & - & - & - & - & - & - & - & - & 1 \\
\hline mortecino & - & - & - & - & - & - & 1 & - & 1 & - & - & - & 2 \\
\hline motilles & - & - & - & - & - & - & - & - & 1 & - & - & - & 1 \\
\hline motita & - & - & - & - & - & - & - & - & - & - & - & 1 & 1 \\
\hline motitas & - & 1 & - & - & - & - & - & - & - & - & - & - & 1 \\
\hline mesach & - & - & 1 & - & - & - & - & - & - & - & - & - & 1 \\
\hline mujercill & - & - & 2 & - & - & - & 3 & - & - & - & - & 1 & 6 \\
\hline mujercillas & - & - & 2 & - & - & - & - & - & - & - & 1 & - & 3 \\
\hline majercitas & - & - & - & - & - & - & 2 & - & - & - & - & 2 & 4 \\
\hline navecica & - & - & - & - & - & - & - & - & 1 & - & - & - & 1 \\
\hline negrilla & - & - & 1 & - & - & - & - & - & - & - & - & - & 1 \\
\hline
\end{tabular}


Anexo 1 (Continuación)

Tabla de las formas diminutivas divididas por obra

\begin{tabular}{|c|c|c|c|c|c|c|c|c|c|c|c|c|c|}
\hline FORMAS & APU & CAM & CAR & $\mathrm{CON}$ & $\cos$ & EXC & FUN & MOD & MOR & POE & REL & VID & TOT \\
\hline negrillo & - & - & - & - & - & - & - & - & - & - & - & 1 & 1 \\
\hline obrillas & - & - & - & - & - & - & - & - & 1 & - & - & - & 1 \\
\hline ocasionciltas & - & - & - & 1 & - & - & - & - & - & - & - & - & 1 \\
\hline ocasioncita & - & - & - & 1 & - & - & - & - & - & - & - & - & 1 \\
\hline ovejitas & - & - & - & - & - & - & 1 & - & - & - & - & - & 1 \\
\hline ovillo & - & - & - & - & - & - & - & - & - & - & - & 1 & 1 \\
\hline pajarillos & - & - & - & - & - & - & - & - & 1 & - & - & - & 1 \\
\hline pajaritos & - & - & - & - & - & - & - & - & - & - & 1 & - & 1 \\
\hline pajecillo & - & - & 1 & - & - & - & - & - & - & - & - & - & 1 \\
\hline pajitas & - & 1 & - & - & - & - & - & - & - & - & - & 2 & 3 \\
\hline palabrilla & - & 1 & - & - & - & - & - & - & - & - & - & - & 1 \\
\hline palabrita & - & - & - & - & - & - & - & - & 1 & - & - & - & 1 \\
\hline patillos & - & - & - & - & - & - & - & - & - & - & 1 & 1 & 2 \\
\hline palmito & - & - & - & - & - & - & - & - & 1 & - & - & - & 1 \\
\hline palomarcitos & - & - & - & - & - & - & 1 & - & - & - & - & - & 1 \\
\hline palomica & - & - & - & - & - & - & - & - & 3 & - & - & - & 3 \\
\hline palomilla & - & - & - & - & - & - & - & - & 1 & - & - & - & 1 \\
\hline palomita & - & - & - & - & - & - & - & - & - & - & - & 1 & 1 \\
\hline papelillo & - & - & 3 & - & - & - & - & - & - & - & - & - & 3 \\
\hline partecita & - & - & - & - & - & 1 & - & - & 1 & - & - & - & 2 \\
\hline pastilles & - & - & 2 & - & - & - & - & - & - & - & - & - & 2 \\
\hline Pastorcicos & - & - & 1 & - & - & - & - & - & - & - & - & - & 1 \\
\hline pastorcillo & - & - & - & 2 & - & - & - & - & - & - & - & - & 2 \\
\hline pastorcillos & - & - & 1 & - & - & - & - & - & 1 & - & - & - & 2 \\
\hline pastorcito & - & 1 & - & - & - & - & 1 & - & - & - & - & - & 2 \\
\hline patecillo & - & - & - & - & - & - & 1 & - & - & - & - & - & 1 \\
\hline patillas & - & - & 3 & - & - & - & - & - & - & - & - & - & 3 \\
\hline pañico & - & - & 1 & - & - & - & - & - & - & - & - & - & 1 \\
\hline pañizueło & - & - & 1 & - & - & - & - & - & - & - & - & - & 1 \\
\hline pecadorcilla & - & 2 & - & - & - & - & - & - & - & - & 1 & 1 & 4 \\
\hline pedacico & - & - & - & - & - & - & - & - & - & - & 1 & - & 1 \\
\hline pedrecillas & - & - & - & - & - & - & - & - & - & - & - & 1 & 1 \\
\hline pelillo & - & - & - & 1 & - & - & - & - & - & - & - & - & 1 \\
\hline pensamientillos & - & - & - & - & - & - & - & - & 1 & - & - & - & 1 \\
\hline pequeñita & - & - & - & 1 & - & - & - & -1 & - & - & - & - & 1 \\
\hline
\end{tabular}


Anexo 1 (Continuación)

Tabla de las formas diminutivas divididas por obra

\begin{tabular}{|c|c|c|c|c|c|c|c|c|c|c|c|c|c|}
\hline FORMAS & APU & CAM & CAR & $\cos \mid$ & $\cos \mid$ & EXC & FuN & MOD & MOR & POE & REL & VID & TOT \\
\hline pequeneiito & - & 1 & - & - & - & - & - & - & - & - & - & - & 1 \\
\hline pequeeiitos & - & - & - & - & - & - & 1 & - & - & - & - & - & 1 \\
\hline Periquito & - & - & 1 & - & - & - & - & - & - & - & - & - & 1 \\
\hline piccecille & - & - & 1 & - & - & - & - & - & - & - & - & - & 1 \\
\hline pobrecica & - & - & - & - & - & - & - & - & 1 & - & - & - & 1 \\
\hline pobrecilla & - & 1 & - & - & - & - & - & - & - & - & - & - & 1 \\
\hline pobrecillas & - & 1 & - & - & - & - & 1 & - & - & - & - & - & 2 \\
\hline pobrecita & - & - & 5 & - & - & - & 1 & - & - & - & - & 1 & 7 \\
\hline pobrecitas & - & 1 & 1 & - & - & - & - & - & - & - & - & 2 & 4 \\
\hline pobrectito & - & 1 & 1 & - & - & - & - & - & - & - & - & 1 & 3 \\
\hline pobrecitos & - & 1 & 1 & - & - & - & - & - & - & - & - & - & 2 \\
\hline polvito & - & - & - & - & - & - & - & - & - & - & - & 1 & 1 \\
\hline poquillo & - & 1 & 1 & - & - & - & - & - & 1 & - & - & 1 & 4 \\
\hline poquita & - & - & - & - & - & - & 1 & - & - & - & - & 1 & 2 \\
\hline poquitas & - & 2 & - & - & - & - & - & - & 1 & - & - & 1 & 4 \\
\hline poquito & - & 13 & 4 & 5 & - & - & 5 & - & 8 & - & - & 9 & 44 \\
\hline poquitos & - & 1 & - & - & - & - & - & - & - & - & - & 1 & 2 \\
\hline portalito & - & - & - & - & - & - & 1 & - & - & - & - & - & 1 \\
\hline prionita & - & - & 1 & - & - & - & - & - & - & - & - & - & 1 \\
\hline prioritus & - & - & 1 & - & - & - & - & - & - & - & - & - & 1 \\
\hline pentica & - & - & - & 1 & - & - & - & - & - & - & - & - & 1 \\
\hline pentito & - & 2 & - & - & - & - & - & - & - & - & - & - & 2 \\
\hline ramillas & - & - & - & - & - & - & - & - & 1 & - & - & - & 1 \\
\hline ramitos & - & - & - & - & - & - & - & - & - & - & - & 1 & 1 \\
\hline natico & - & - & 1 & - & - & - & - & - & - & - & - & - & 1 \\
\hline regalitio & - & - & 1 & - & - & - & - & - & - & - & - & - & 1 \\
\hline rinconcito & - & - & - & - & - & - & - & - & - & - & - & 2 & 2 \\
\hline sabandijita & - & - & 1 & - & - & - & - & - & - & - & - & - & 1 \\
\hline suntica & - & - & 1 & - & - & - & - & - & - & - & - & - & 1 \\
\hline sentico & - & - & 1 & - & - & - & - & - & - & - & - & - & 1 \\
\hline santita & - & - & 2 & - & - & - & - & - & - & - & - & - & 2 \\
\hline santito & - & - & 1 & - & - & - & - & - & - & _- & - & - & 1 \\
\hline sobrinito & - & - & 1 & - & - & - & - & - & - & - & - & - & 1 \\
\hline solilin & - & - & 1 & - & - & - & - & - & - & - & - & - & 1 \\
\hline suspensioncilla & - & -1 & - & - & - & -1 & - & -1 & 1 & -1 & - & - & 1 \\
\hline
\end{tabular}


Anexo 1 (Continuación)

Tabla de las formas diminutivas divididas por obra

\begin{tabular}{|l|c|c|c|c|c|c|c|c|c|c|c|c|c|}
\hline \multicolumn{1}{|c|}{ FORMAS } & APU & CAM & CAR & CON & COS & EXC & FUN & MOD & MOR & POE & REL & VID & TOT \\
\hline tamanita & - & - & 1 & - & - & - & - & - & - & - & - & - & 1 \\
tantico & - & - & 2 & - & - & - & - & - & - & - & - & 2 & 4 \\
tantito & - & 2 & - & - & - & - & - & - & 1 & - & 2 & 5 & 10 \\
tejuelos & - & - & 1 & - & - & - & - & - & - & - & - & - & 1 \\
temorcillos & - & - & - & 1 & - & - & - & - & - & - & - & 1 & 2 \\
Teresica & - & - & 7 & - & - & - & - & - & - & - & - & - & 7 \\
Teresita & - & - & 3 & - & - & - & - & - & - & - & - & - & 3 \\
termuritas & - & - & 1 & - & - & - & - & - & - & - & - & - & 1 \\
tortolito & - & - & - & - & - & - & - & - & 1 & - & - & - & 1 \\
trabajillo & - & - & - & - & - & - & - & - & 1 & - & - & - & 1 \\
trabajillos & - & - & - & - & - & - & 1 & - & 1 & - & - & - & 2 \\
tristecilla & - & - & 2 & - & - & - & - & - & - & - & - & - & 2 \\
tropiecillos & - & - & - & 1 & - & - & - & - & - & - & - & - & 1 \\
ungüentillo & - & - & 1 & - & - & - & - & - & - & - & - & - & 1 \\
varillas & 3 & - & - & - & - & - & - & - & - & - & - & - & 3 \\
vejecuel & - & - & 2 & - & - & - & - & - & - & - & - & - & 2 \\
ventanil. & 1 & - & - & - & - & - & - & 1 & - & - & - & 1 & 3 \\
ventanillas & 2 & - & - & - & - & - & 1 & - & - & - & - & - & 3 \\
viejecita & - & - & - & - & - & - & - & - & - & - & - & 1 & 1 \\
\hline TOTALES & 8 & 48 & 191 & 24 & 1 & 2 & 48 & 2 & 61 & 6 & 11 & 78 & 480 \\
\hline
\end{tabular}

Anexo 2

Códigos de las obras

1. VID LIBRO DE LA VIDA

2. CAM CAMINO DE PERFECCIÓN

3. FUN LIBRO DE LAS FUNDACIONES

4. CAR 'EPISTOLARIO

5. MOR MORADAS DEL CASTILLO INTERIOR

6. REL RELACIONES

7. CON CONCEPTOS DEL AMOR DE DIOS

8. EXC EXCLAMACIONES 

9. COS CONSTITUCIONES
10. MOD MODO DE VISITAR LOS CONVENTOS
11. POE POESÍAS
12. APU APUNTACIONES
13. RES RESPUESTA A UN DESAFIO
14. VEJ VEJAMEN

\section{NOTAS BIBLIOGRÁFICAS}

A. Saba, D. Ratti, M. N. Catarsi, G. Cappelli, Análisis morfosintáctico y lematización de textos en lengua española, in «Ordenadores y lengua española» CATARSI, M.N., RATTI, D., SABA, A., SASSI, M., (Eds.), Giardini, Pisa, pp. 97 106, 1982.

A. Saba, D. Ratti, M. N. Catarsi, G. Cappelli, Morfsin: Analizzatore Morfosintattico di testi in lingua spagnola, ILC-ALN-1986-1, Pisa, 1986.

A. SABA, M. N. CATARSI, Lemmatizzazione manuale e lemmatizzazione automatica: problemi e proposte, ILC-ALN-1987-3, Pisa, 1987.

E. CARPI, A. SABA, M. SASSI, Concordanze del *Libro de la Vida* di Teresa de Ávila, Servizio Editoriale D.S.U., Pisa, 1987.

E. CARPI, A. SABA, M. SASSI, Exclamaciones del alma a Dios. Concordanze per lemma e Repertorio delle forme enfatiche nell'opera omnia di Teresa de Ávila, Servizio Editoriale D.S.U., Pisa, 1992.

E. CARPI, A. SABA, M. SASSI, Archivo lematizado informatizado de la Obra completa de Teresa de Avila. ILC-ALN 1995.

E. CARPI, A. SABA, M. SASSI, L'uso del diminutivo nell'opera di Santa Teresa de Avila. S.T.A.R. (Servizio Tecnografico Area di Ricerca del CNR), Febbraio 1995 , Pisa. 